\title{
Off-lattice model for the phase behavior of lipid-cholesterol bilayers
}

Nielsen, Morten; Miao, Ling; Ipsen, John Hjorth; Zuckermann, Martin; Mouritsen, Ole G.

Published in:

Physical Review E. Statistical, Nonlinear, and Soft Matter Physics

Link to article, DOI:

10.1103/PhysRevE.59.5790

Publication date:

1999

Document Version

Publisher's PDF, also known as Version of record

Link back to DTU Orbit

Citation (APA):

Nielsen, M., Miao, L., Ipsen, J. H., Zuckermann, M., \& Mouritsen, O. G. (1999). Off-lattice model for the phase behavior of lipid-cholesterol bilayers. Physical Review E. Statistical, Nonlinear, and Soft Matter Physics, 59(5), 5790-5803. https://doi.org/10.1103/PhysRevE.59.5790

\section{General rights}

Copyright and moral rights for the publications made accessible in the public portal are retained by the authors and/or other copyright owners and it is a condition of accessing publications that users recognise and abide by the legal requirements associated with these rights.

- Users may download and print one copy of any publication from the public portal for the purpose of private study or research.

- You may not further distribute the material or use it for any profit-making activity or commercial gain

- You may freely distribute the URL identifying the publication in the public portal 


\title{
Off-lattice model for the phase behavior of lipid-cholesterol bilayers
}

\author{
Morten Nielsen, ${ }^{1}$ Ling Miao, ${ }^{2}$ John H. Ipsen, ${ }^{2}$ Martin J. Zuckermann,,${ }^{1, *}$ and Ole G. Mouritsen ${ }^{2}$ \\ ${ }^{1}$ Centre for the Physics of Materials, Department of Physics, McGill University, 3600 University Street, Montreal, Canada H3A 2T8 \\ ${ }^{2}$ Department of Chemistry, Technical University of Denmark, Lyngby, Denmark
}

(Received 6 November 1998)

\begin{abstract}
Lipid bilayers exhibit a phase behavior that involves two distinct, but coupled, order-disorder processes, one in terms of lipid-chain crystalline packing (translational degrees of freedom) and the other in terms of lipidchain conformational ordering (internal degrees of freedom). Experiments and previous approximate theories have suggested that cholesterol incorporated into lipid bilayers has different microscopic effects on lipid-chain packing and conformations and that cholesterol thereby leads to decoupling of the two ordering processes, manifested by a special equilibrium phase, "liquid-ordered phase," where bilayers are liquid (with translational disorder) but lipid chains are conformationally ordered. We present in this paper a microscopic model that describes this decoupling phenomena and which yields a phase diagram consistent with experimental observations. The model is an off-lattice model based on a two-dimensional random triangulation algorithm and represents lipid and cholesterol molecules by hard-core particles with internal (spin-type) degrees of freedom that have nearest-neighbor interactions. The phase equilibria described by the model, specifically in terms of phase diagrams and structure factors characterizing different phases, are calculated by using several Monte Carlo simulation techniques, including histogram and thermodynamic reweighting techniques, finitesize scaling as well as non-Boltzmann sampling techniques (in order to overcome severe hysteresis effects associated with strongly first-order phase transitions). The results provide a consistent interpretation of the various phases of phospholipid-cholesterol binary mixtures based on the microscopic dual action of cholesterol on the lipid-chain degrees of freedom. In particular, a distinct small-scale structure of the liquid-ordered phase has been identified and characterized. The generic nature of the model proposed holds a promise for a unifying description for a whole series of different lipid-sterol mixtures. [S1063-651X(99)09305-8]

PACS number(s): 05.70.Fh, 64.60.Cn, 83.20.Jp, 87.16.-b
\end{abstract}

\section{INTRODUCTION}

Hydrated bilayers composed of amphiphilic lipid molecules have attracted a remarkable amount of interest from both the biological and the physics communities, because of their biological significance as one of the basic architectural components in biological cell membranes, and also because of their physical significance as model systems that offer both a richness and a complexity of physical phenomena [1]. From the physical point of view, each monolayer in a lipid bilayer constitutes a two-dimensional (2D) system of interacting lipid molecules, the hydrocarbon chains of which can be thermally excited to a large number of conformational states. Naturally, a full physical description of a lipid bilayer must involve at least two types of fundamental degrees of freedom intrinsic in the bilayer: translational degrees of freedom, and "internal" molecular degrees of freedom that correspond to the chain conformational states. At the microscopic level, the interplay between these two types of degrees of freedom can be clearly and simply defined in terms of specific microscopic interactions and molecular mechanisms. But at the macroscopic level, the interplay can manifest itself intricately in the ordering processes in a lipid bilayer, depending on the nature of the microscopic interactions or the molecular mechanisms. Our previous work [2] concentrated on the issue of how details of microscopic interactions affect the macroscopic coupling between the two

\footnotetext{
*Author to whom correspondence should be addressed. Electronic address: martin@physics.mcgill.ca
}

types of degrees of freedom in models for single-component lipid bilayers. Our present paper continues on the same general theme of the interplay between the translational and the internal degrees of freedom, but focuses on investigating the effects of introducing into a one-component system a second species of "model molecules" that display a duality in their interactions with the translational and the internal degrees of freedom of the native molecules, mimicking, in fact, the molecular mechanisms of cholesterol molecules in lipid bilayers (see below).

The existence of two principal thermodynamic phases of a lipid-bilayer system has been well established. These are characterized by different types of macroscopic behavior of both the translational and the chain conformational degrees of freedom: a low-temperature gel phase, which is a 2D solid phase with (quasi-) long-range translational order and which also has a high degree of collective ordering in chain conformations of the lipid molecules in the system, and a hightemperature liquid-crystalline phase, which displays macroscopic disorder in both the translational (as does a liquid) and the chain conformational degrees of freedom. Although the common terminology in biophysical literature describes the two phases as the gel phase and the liquid-crystalline phase, we for our purposes label the two phases with so (for solid, chain ordered) and ld (for liquid, chain disordered), respectively. It can be concluded from experimental data that, thermally driven, the translational order and the chain conformational order that are characteristic of the so phase disappear or appear simultaneously at a particular temperature called the temperature of the "main transition," in almost all of the systems of one-component lipid bilayers stud- 
ied [3]. In other words, the translational and the chain conformational degrees of freedom appear macroscopically coupled.

However, there are no fundamental physical principles dictating that this macroscopic coupling be the necessary generic feature of the thermodynamic behavior of either twodimensional systems with both translational and internal degrees of freedom in general, or lipid bilayers in particular. Indeed, our previous study of minimal models for $2 \mathrm{D}$ random (off-lattice) Ising systems and one-component lipid bilayers has shown that it is only a matter of engineering microscopic interactions to macroscopically decouple the two types of degrees of freedom [2]. An intermediate phase does exist as part of the generic phase behavior of systems (such as lipid bilayers) with both the translational and internal degrees of freedom: a phase where the (quasi-) long-range translational order is broken, but where the internal (chain conformational) degrees of freedom remain macroscopically ordered. We referred to this intermediate phase as the lo (for liquid, chain ordered) phase. We note here, however, that concrete experimental evidence has yet to be found for the existence of such a phase in one-component lipid bilayers, although some evidence has been found for lipid monolayers [4].

The lo phase has been discovered, however, in bilayer systems of some lipid-cholesterol binary mixtures. Cholesterol is an important molecular constituent of the membranes of eukaryotic cells and it is mostly distributed in the cell plasma membrane. The question of the biological relevance of cholesterol has intrigued many researchers, as evidenced by a large body of both theoretical and experimental work on lipid-cholesterol bilayers and monolayers [5-10], among which we briefly review those that are most relevant to the purpose of our study. The evidence for the existence of the lo phase in bilayers of dimyristoyl phosphatidylcholine (DMPC)-cholesterol mixtures was first given by Needham, McIntosh, and Evans on the basis of their micromechanical measurements [9]. A full phase diagram in terms of cholesterol concentration and temperature was firmly established for bilayers of dipalmitoyl phosphatidylcholine (DPPC)cholesterol mixtures by Vist and Davis by combining data from deuterium-NMR, differential scanning calorimetry and electron spin resonance studies [10]. This phase diagram displays a modest depression of the main-transition (so-ld) temperature for low concentrations of cholesterol; and remarkably, it demonstrates a macroscopic decoupling of the translational and the chain conformational degrees of freedom, as manifested by a high-temperature ld-lo coexistence and a low-temperature so-lo coexistence at intermediate concentrations of cholesterol as well as a single lo phase region at high cholesterol concentrations.

Molecular mechanisms underlying the apparent ability of cholesterol to decouple the macroscopic processes of lateral ordering and chain ordering in a lipid bilayer were investigated in a theoretical study based on a lattice model [5]. In the model, each lattice site was assigned a multivalued variable corresponding to chain conformational states. The physics governing this set of degrees of freedom was described by a multistate (Pink) model [11]. In addition, a multistate Potts model was employed to give, in a very approximate way, a phenomenological account of the lateral crystalliza- tion (ordering) process, describing the process only in terms of thermal energetics of the grain boundaries [12]. The final essential ingredient of the model was a hypothesis of a dual molecular mechanism of cholesterol: on the one hand, a cholesterol molecule acts as an "ice breaker," described in the model as a substitutional impurity that weakens the interlipid interactions responsible for crystallization; on the other hand, the molecule acts as a "chain rigidifier," tending to induce its neighboring lipid chains into conformationally ordered states. Calculations based on a mean-field theory of the model predicted a phase diagram of DPPC-cholesterol bilayers that agreed qualitatively with the experimental phase diagram. This model study certainly provided concrete theoretical support for the hypothesis and strongly suggested that the lattice model may have captured some of the essence of the microscopic physics governing the thermodynamic phase behavior of lipid-cholesterol bilayers. This model, however, suffers a fundamental shortcoming inherent in the lattice description: the description of the translational degrees of freedom and the microscopic physics underlying the associated ordering process is not really realistic.

Motivated by the phenomenology and the understanding established by the previous experimental and theoretical work on lipid-cholesterol systems, given the context of our previous work of investigating macroscopic interplay between translational and internal degrees of freedom in twodimensional systems within a minimal model, and equipped with a computer-simulation algorithm that we developed for the purpose of providing a full description of translational degrees of freedom, we have found it natural to extend our previous minimal model for one-component lipid bilayers by including a second species of particles that model the dual molecular mechanism of cholesterol molecules in lipid bilayers. Our focus is, therefore, on exploring the generic thermodynamic consequences of the cholesterol-like molecular mechanism, in terms of the macroscopic ordering processes associated with the two types of degrees of freedom. In the same spirit of our earlier work, our current model for the cholesterol-like particle is also designed to be minimal, in that it contains a very small number of model parameters, but still reflects the dual molecular function of the particle of being both an "ice breaker" and a "chain rigidifier." As the results of our study will demonstrate below, certain kinds of our model particles, corresponding to a range of detailed models of their dual-natured coupling to the "lipid molecules,' do indeed macroscopically decouple the translational and internal degrees of freedom, leading to the separate appearance of two distinct ordering processes and the existence of a lo-type phase. It is perhaps useful in guiding our presentation and discussion to make note at this point. Although we often refer to the particles in our model as "lipid molecules" and "cholesterol molecules" and to the systems as "lipid bilayers," we are fully aware that our descriptions of the microscopic properties of our "model molecules" are considerably simplified pictures of those of real lipid and cholesterol molecules, as our emphasis is on revealing generic physics rather than on providing quantitative interpretations for the experiments. And the generic physics we are concerned with should be viewed in a broader context: it may well also be relevant to certain two-dimensional nonlipid systems where both translational and internal molecular 
degrees of freedom are thermodynamically relevant.

The plan for the presentation of the rest of the paper is as follows. In Sec. II, we first review briefly both the randomlattice algorithm and the essential results from our previous study of the macroscopic coupling between the translational and the chain conformational degrees of freedom in onecomponent systems. We then give a basic description of the extended microscopic model for a "lipid-cholesterol" bilayer, which defines the microscopic interactions between the lipid molecules and between the lipid and cholesterol molecules and highlights the dual function of cholesterol molecules. In Sec. III, we discuss different numerical methods that were used in the Monte Carlo simulations of the extended model, with a particular reference to the use of an effective-Hamiltonian technique that reduces the problems associated with the strong hysteresis frequently encountered in our simulations. In Sec. IV, we present the results of the Monte Carlo simulations of the extended model. These results consist of a detailed "lipid-cholesterol" phase diagram obtained from simulations based on the techniques discussed in Sec. III as well as characterizations of lateral structures in the different thermodynamic phases. Finally, in Sec. V we examine our results in the light of earlier research on lipidcholesterol systems and discuss their significance, in particular, the applicability of the calculated phase diagram. We also propose possible modifications or extensions of our present model, which may be applied to bilayer systems of binary mixtures of lipids with sterol molecules other than cholesterol.

\section{MODEL}

As stated in the preceding section, the microscopic model to be discussed in the present paper is an extension of a model proposed earlier to describe the generic phase behavior of single-component lipid bilayers [2]. Hence, we first give a summary of the details of that model and the principal results from the computer-simulation study of the model. This earlier model gives a minimal description of the microscopic physics governing the phase behavior of lipid bilayers, in terms of two different types of fundamental degrees of freedom-the internal degrees of freedom representing chain conformations and the translational degrees of freedom describing the positions of the particles. Two distinct states form the phase space of each conformational variable: an ordered (o) state, corresponding to an all-trans configuration, and a disordered (d) state, modeling excited states where lipid chains become conformationally disordered due to trans-gauche isomerization. The ordered state is a nondegenerate ground state while the disordered state is characterized by an excitation energy and a high degeneracy representing the large number of possible conformational states of transgauche isomerizations [13]. A genuine representation of the configuration space associated with the translational variables is achieved through a specially designed 2D randomlattice simulation algorithm. A brief description of this algorithm will be given in Sec. III.

The two types of degrees of freedom are microscopically coupled through pairwise interaction potentials, which depend on both the conformational states and the interparticle distance of two interacting particles. Explicitly, the following
Hamiltonian defines the microscopic model:

$$
H_{0}=\sum_{i} E_{d} \mathcal{L}_{i d}+\sum_{\langle i<j\rangle} V_{o-o}(R) \mathcal{L}_{i o} \mathcal{L}_{j o}+\Pi A .
$$

Here $\langle i<j\rangle$ denotes a summation over nearest neighbors and $i$ is an index labeling the particles in the system. $\mathcal{L}_{i d}$ and $\mathcal{L}_{i o}$ are occupation variables that are unity when the $i$ th particle is in the ordered and the disordered states, respectively, and zero otherwise. $E_{d}$ is the excitation energy of the disordered conformational state and $V_{o-o}(R)$ is a distance-dependent interaction potential between two neighboring particles that are both in the ordered state. $\Pi$ is, in effect, a lateral surface pressure stabilizing the system against lateral expansion and $A$ is the total area of the system. The interaction potential $V_{o-o}(R)$ is composed of a hard-core repulsive potential and two attractive square-well potentials, as illustrated in Fig. 1(a), being a simple model of a realistic short-range molecular interaction.

The main results of our previous simulation study of this model are summarized in Fig. 2, a phase diagram given in terms of temperature and a parameter $V_{0} / J_{0}$ measuring the relative strength of the two square-well attractions. The point of key importance in this phase diagram is the appearance of two distinct regimes, separated by a triple point, of different types of macroscopic interplay between the two types of degrees of freedom. A regime of macroscopic coupling between the two types of degrees of freedom, as observed in most systems of pure lipid bilayers, exists for values of $V_{0} / J_{0}$ greater than the triple-point value; however, a regime of macroscopic decoupling also exists as part of the generic thermodynamic behavior of the model, for values of $V_{0} / J_{0}$ smaller than the triple-point value, where two distinct ordering transitions take place successively, separated by an intermediate lo phase. In order to highlight the influence of the cholesterol-like molecular mechanism as proposed in the Introduction, we choose in the present paper to concentrate on the regime where the two sets of degrees of freedom are macroscopically coupled in the absence of cholesterol.

Based upon the model and the physics described above, a minimal model, which describes a system containing both lipid chain particles and cholesterol molecules, can be built by adding additional microscopic interaction terms to the Hamiltonian in Eq. (1):

$$
H=H_{0}+H_{o-d}+H_{o-c}+H_{d-c}+H_{c-c} .
$$

Here $H_{o-d}, H_{o-c}, H_{d-c}$, and $H_{c-c}$, as indicated by the different subscripts, represent the pairwise interaction potentials between an ordered chain and a disordered chain, an ordered chain and a cholesterol molecule, a disordered chain and a cholesterol molecule, and two cholesterol molecules, respectively; they are defined as follows:

$$
\begin{aligned}
& H_{o-d}=\sum_{\langle i<j\rangle} V_{o-d}\left(R_{i j}\right)\left\{\mathcal{L}_{i o} \mathcal{L}_{j d}+\mathcal{L}_{j o} \mathcal{L}_{i d}\right\}, \\
& H_{o-c}=\sum_{\langle i<j\rangle} V_{o-c}\left(R_{i j}\right)\left\{\mathcal{L}_{i o} \mathcal{L}_{j c}+\mathcal{L}_{j o} \mathcal{L}_{i c}\right\},
\end{aligned}
$$


(a)

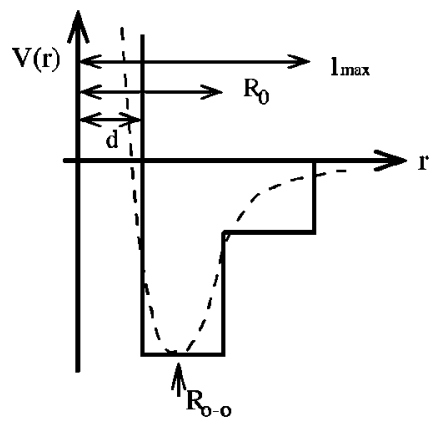

(c)

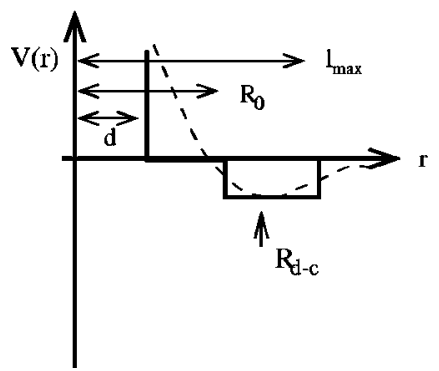

(b)

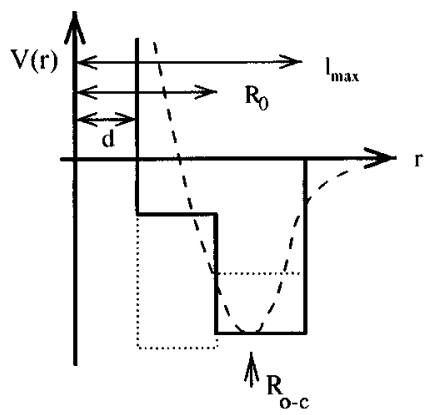

(d)

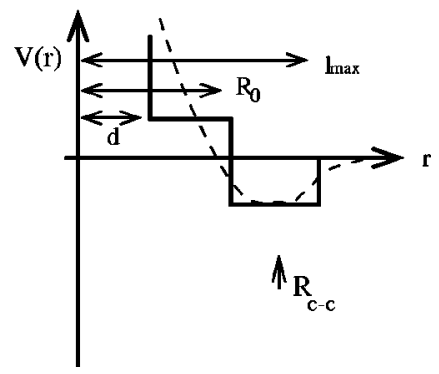

FIG. 1. Model interaction potentials. (a) $V_{o-o}(R), \quad(\mathrm{b}) V_{o-c}(R), \quad(\mathrm{c}) V_{d-c}(R)$, and (d) $V_{c-c}(R) . d$ is the hard-core radius, $R_{0}$ is the radius of the short-range square-well potential and $l_{\text {max }}$ is the range of the longer range square-well potential. The ratio $R_{0} / d$ is chosen so that $R_{o-c} / R_{o-o} \approx 1.3$, making the average surface area of the cholesterol molecules about 30\% larger than that of the lipid chains in the ordered state.

$$
\begin{gathered}
H_{d-c}=\sum_{\langle i<j\rangle} V_{d-c}\left(R_{i j}\right)\left\{\mathcal{L}_{i d} \mathcal{L}_{j c}+\mathcal{L}_{j d} \mathcal{L}_{i c}\right\}, \\
H_{c-c}=\sum_{\langle i<j\rangle} V_{c-c}\left(R_{i j}\right)\left\{\mathcal{L}_{i c} \mathcal{L}_{j c}\right\},
\end{gathered}
$$

where an additional occupation variables $\mathcal{L}_{i c}$ is introduced to account for the presence of the cholesterol molecules in the system. $\mathcal{L}_{i c}$ is unity if the $i$ th particle is a cholesterol molecule and is zero otherwise. The energy of interaction between two chains both in the disordered state is set to zero. For completeness, the model of Eq. (2) includes a term for the interaction between an ordered and a disordered lipid chain. This term was not included in the Hamiltonian in Eq. (1) since it has no importance for the generic phase behavior of the model.

Reflecting our strategy of performing minimal modeling, the additional microscopic interactions, $V_{o-c}(R), V_{d-c}(R)$, $V_{c-c}(R)$, and $V_{o-d}(R)$, are each approximated, similarly as $V_{o-o}(R)$ in $H_{0}$, by a sum of a hard-core repulsive potential of range $d$, a short-range square-well potential $V^{S}(R)$,

$$
V^{s}(R)=\left\{\begin{array}{l}
-V^{s}, d<R \leqslant R_{0} \\
0 \text { otherwise, }
\end{array}\right.
$$

and a longer-range attractive square-well potential $V^{l}(R)$,

$$
V^{l}(R)=\left\{\begin{array}{l}
-V^{l}, \quad d<R \leqslant l_{\max } \\
0 \text { otherwise. }
\end{array}\right.
$$

Some of the microscopic interactions are sketched in Fig. 1 to illustrate our specific way of modeling the dual molecular function of a cholesterol molecule. A comparison of Fig. 1(a) and Fig. 1(b) illustrates the "ice-breaker' mechanism, as the interactions involved imply that a cholesterol molecule dissolved in an ordered-chain environment tends to have a larger surface area than that of a lipid chain, thus disrupting the lateral packing of the ordered chains. Similarly, a comparison of Fig. 1(b) and Fig. 1(c) makes the "chainrigidifier' mechanism clear, as the given interactions simply mean that a cholesterol molecule prefers its neighboring chains to be in the conformationally ordered state.

\section{SIMULATION METHODS}

Before presenting the numerical results obtained from the simulation studies of the microscopic model of Eq. (2) we describe the statistical mechanical formulation of the prob-

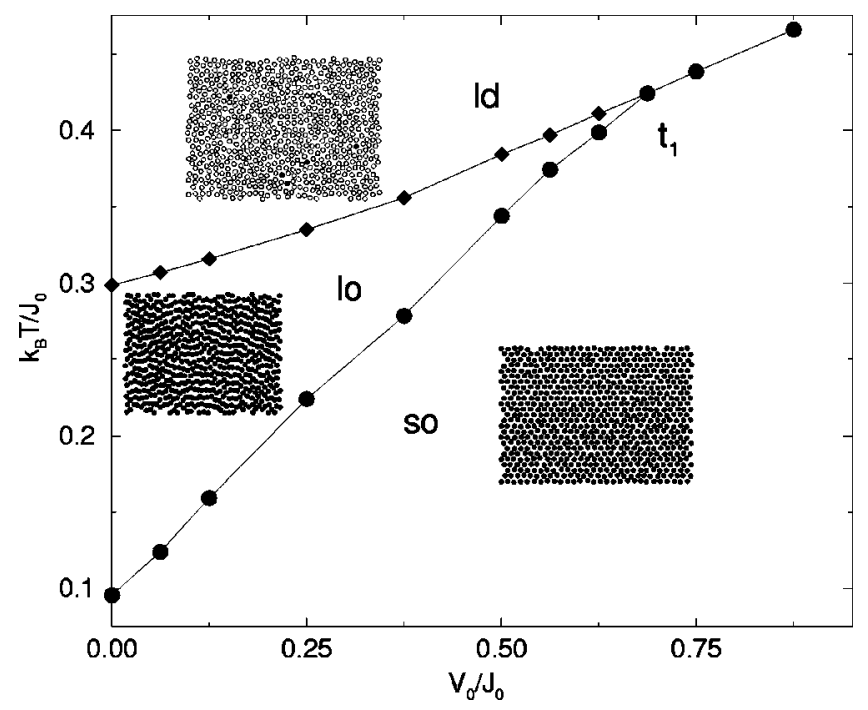

FIG. 2. Phase diagram for the model of the pure lipid bilayer system. All three phase boundaries are first-order phase boundaries. The insets show snapshots of typical microconfigurations for the three different phases labeled so (solid-ordered), ld (liquiddisordered), and lo (liquid-ordered), respectively. Chains in the disordered state are plotted as $(O)$ and chains in the ordered chain state as (-). The three snapshots are not given to scale. $t_{1}$ is the triple point described in the text. 
lem in terms of thermodynamic ensembles and simulation procedures, and discuss in some detail the simulation methods that have been employed in order to establish a coherent picture of the thermodynamic behavior of the model.

The system under consideration consists of a total number of $N$ particles, including both "lipid" and "cholesterol" particles. All simulations performed used the Monte Carlo Metropolis algorithm [14], for fixed $N, T$, and surface pressure $\Pi$.

Two different thermodynamic ensembles have been used regarding the molecular composition of the system: (a) a semigrand canonical ensemble, in which the number of "cholesterol" particles is controlled by a parameter $\mu_{\Delta}$ representing the difference between the chemical potentials of "lipid" and "cholesterol" particles, and, therefore, fluctuates and (b) a canonical ensemble, in which the number of "cholesterol" particles, i.e., the "cholesterol" concentration $x_{c}$ is fixed. The two formulations are complementary to each other. The simulations formulated based on the semigrand canonical ensemble straightforwardly (at least in principle) yield as functions of $T$ and $\mu_{\Delta}$ equilibrium probability distributions for relevant quantities such as the "cholesterol" concentration. Hence, the phase diagram of the system, both in the $\left(\mu_{\Delta}, T\right)$ representation and in the $\left(x_{c}, T\right)$, can be unambiguously obtained from the probability distributions.

Our simulation algorithm must explore the entire phase space of the system, which is a superposition of the subspace spanned by the translational degrees of freedom and that spanned by the conformational degrees of freedom as well as, when the semigrand canonical ensemble is used, the subspace associated with compositional fluctuations. Accessing the phase space associated with the conformational degrees of freedom is straightforward as it only involves random updating of conformational states of all "lipid" particles. Similarly, compositional fluctuations are represented by random attempts to exchange each particle in the system with a particle from either of the "lipid" and "cholesterol" reservoirs. The nonconventional element of our simulation algorithm is a random-lattice algorithm developed to deal with the translational degrees of freedom [2]. The details concerning the implementation of this random-lattice algorithm have been published in Ref. [2]; only the essentials are given here. The algorithm employs a network (random-lattice) representation of spatial configurations of a 2D dense system of many particles and generates the phase space of spatial configurations by performing three basic steps: (a) movements of the particles, (b) changes in local connectivity of the network, and (c) changes in the overall area of the system. The combination of steps (a) and (b) ensures the occurrence of both lateral diffusion of the particles and fluctuations in local particle distributions (local densities) that are the necessary manifestations of the translational invariance inherent in any general microscopic description of a 2D liquid system and step (c) allows for changes in global density of the system. In order to ensure detailed balance in the simulations, or the symmetry of the Markov chain generated by the algorithm, all of the different updating procedures have been combined in a random manner. In other words, the simulation algorithm does not impose any preferred sequential order in the procedures that update different types of degrees of freedom [15]. One complete Monte Carlo step (MCS), a time unit for the simulations, is defined to be the time needed to perform on average one complete pass through steps (a), (b), and (c), and one complete run through changing the molecular type or conformational state of each particle in the system.

A straightforward implementation of the updating procedures outlined in the previous paragraph leads to a serious difficulty when investigating the thermodynamic transitions between different phases, especially the transitions between so and Id phases. The difficulty is essentially due to the presence of a very strong hysteresis. There are several reasons underlying the strong hysteresis. A simulated process during which the system passes through a region of barrier between two coexisting equilibrium phases corresponds to a sequence of concerted simulation steps. However, each simulation step in the algorithm involves only a single molecule and small variations in microscopic configurations, or the microscopic energy of the system. Given the already large phase space associated with a single molecule and an increase in the microscopic energy corresponding to the barrier height, each step in the barrier-crossing sequence can only be tried with a small probability $p_{0}$ and realized with an even smaller probability $p_{0} e^{-\beta \Delta E_{1}}$, where $\Delta E_{1}$ here represents the energy increase in a single simulation step. Crossing a high or even modest energy barrier, $\Delta E$, therefore implies a very long sequence of simulation steps and is dictated by a total probability of roughly $\left(p_{0}\right)^{M} e^{-\beta \Delta E}$. This probability is clearly rendered very small by the large number of steps in the sequence $M$. For a modest height of an energy barrier of $4 k_{B} T$, one barrier crossing from an so state to an ld state requires typically $10^{7}$ MCS's for a system of linear size $L=12$. This difficulty makes it practically impossible to reliably identify phase transitions.

Part of the difficulty, which is due to a very small $\left(p_{0}\right)^{M}$, is inherent in the algorithm and, therefore, cannot be removed unless some special algorithms are designed and used. However, the part of the difficulty that arises from the energy barrier can be overcome by employing certain techniques. We have used simulation techniques based on a modified Hamiltonian. This method exploits the idea of developing an "artificial" Hamiltonian that yields a considerably diminished energy barrier. The equilibrium distribution functions for the original Hamiltonian can then be established from the alternative simulations of the modified Hamiltonian through a simple reweighting relation. This method can be considered as a generalization of other Monte Carlo sampling schemes such as the multicanonicalensemble scheme [16]. For a complete discussion of the method and related references, the reader is referred to Ref. [17].

In our simulation study of the microscopic model, Eq. (2), this method has been implemented in simulations performed within the semigrand canonical ensemble. One of the most important equilibrium distribution functions is $\mathcal{P}\left(T, \mu_{\Delta} ; \varepsilon, x_{c}\right)$, where $T$ and $\mu_{\Delta}$ are the thermodynamic control parameters, and $\varepsilon \equiv E / N$ and $x_{c}$ are the energy per particle and cholesterol concentration, respectively. A corresponding spectral free-energy function can now be defined as $\mathcal{F}\left(\varepsilon, x_{c}\right)=-k_{B} T \ln \mathcal{P}\left(\varepsilon, x_{c}\right)$, which displays a barrier when coexistence conditions corresponding to special values of $T$ and $\mu_{\Delta}$ are approached. Given an original Hamiltonian $H$, a 
natural candidate for a modified Hamiltonian can have the following functional form,

$$
\bar{H}=H+f(\varepsilon),
$$

where $f(\varepsilon)$, known as the shape function, must be chosen in such a way that the spectral free-energy function corresponding to the modified Hamiltonian shows no significant barrier, as described below. Straightforward simulations of the modified Hamiltonian yield a modified probability distribution function, $\overline{\mathcal{P}}\left(\varepsilon, x_{c}\right)$, which, when normalized, has a simple relationship to the original $\mathcal{P}\left(\varepsilon, x_{c}\right)$,

$$
\overline{\mathcal{P}}\left(\varepsilon, x_{c}\right)=\frac{\mathcal{P}\left(\varepsilon, x_{c}\right) e^{-\beta f(\varepsilon)}}{\sum_{\varepsilon^{\prime}, x_{c}^{\prime}} \mathcal{P}\left(\varepsilon^{\prime}, x_{c}^{\prime}\right) e^{-\beta f\left(\varepsilon^{\prime}\right)}} .
$$

$\mathcal{P}\left(\varepsilon, x_{c}\right)$ is hence easily established from $\overline{\mathcal{P}}\left(\varepsilon, x_{c}\right)$, based on the above equation and the normalization of $\overline{\mathcal{P}}\left(\varepsilon, x_{c}\right)$ :

$$
\mathcal{P}\left(\varepsilon, x_{c}\right)=\frac{\overline{\mathcal{P}}\left(\varepsilon, x_{c}\right) e^{\beta f(\varepsilon)}}{\sum_{\varepsilon^{\prime}, x_{c}^{\prime}} \overline{\mathcal{P}}\left(\varepsilon^{\prime}, x_{c}^{\prime}\right) e^{\beta f\left(\varepsilon^{\prime}\right)}},
$$

which leads to

$$
\begin{aligned}
& \mathcal{P}\left(x_{c}\right)=\sum_{\varepsilon^{\prime}} \mathcal{P}\left(\varepsilon^{\prime}, x_{c}\right), \\
& \mathcal{P}(\varepsilon)=\sum_{x_{c}^{\prime}} \mathcal{P}\left(\varepsilon^{\prime}, x_{c}\right) .
\end{aligned}
$$

A judicious choice for $f(\varepsilon)$ requires some prior knowledge of the original spectral function $\mathcal{F}(\varepsilon)=-k_{B} T \ln \mathcal{P}(\varepsilon)$; such knowledge can be obtained from simulations of systems of very small sizes. Establishing the phase diagram, especially the loci of phase coexistence, needs sufficient data from systematic simulations of systems of larger sizes. Iteration of a five-step procedure accomplishes the task:

(i) At values of $T$ and $\mu_{\Delta}$ estimated to be close to true coexistence conditions, an initial estimate of $\mathcal{F}(\varepsilon)$ is obtained from a simulation of a system of a relatively small size $L$ governed by the original Hamiltonian $H$, and is denoted $\mathcal{F}_{L}(\varepsilon)$.

(ii) An extrapolation based on the size dependence of the energy barrier is used to approximate the barrier of a system of a larger size $L^{\prime}$ and in turn, the shape function:

$$
f(\varepsilon)=-\mathcal{F}_{L^{\prime}}(\varepsilon)=-\frac{L^{\prime}}{L} \mathcal{F}_{L}(\varepsilon)
$$

when $\varepsilon$ lies in the barrier region. $f(\varepsilon)$ then defines the modified Hamiltonian, which is used in a second simulation of a system of size $L^{\prime}$. From this simulation, the modified probability distribution function, $\overline{\mathcal{P}}_{L^{\prime}}\left(\varepsilon, x_{c}\right)$, is obtained.

(iii) $\mathcal{P}_{L^{\prime}}\left(\varepsilon, x_{c}\right)$ is reconstructed from $\overline{\mathcal{P}}_{L^{\prime}}\left(\varepsilon, x_{c}\right)$ by use of Eq. (8).

(iv) Based on $\mathcal{P}_{L^{\prime}}\left(\varepsilon, x_{c}\right)$, the Ferrenberg-Swendsen reweighting technique [18] is applied in order to obtain a better estimate of the coexistence condition and an improved approximation of $\mathcal{F}(\varepsilon)$ at the coexistence by $\mathcal{F}_{L^{\prime}}(\varepsilon)$.

(v) If desired, another iteration is started from step (ii) with $\mathcal{F}_{L^{\prime}}(\varepsilon)$, either to obtain an improved statistical sampling of $\mathcal{F}_{L^{\prime}}\left(\varepsilon, x_{c}\right)$ for the same system size or to simulate a larger system.

In the simulations, a system in a laterally disordered state is prepared at a high temperature. The system is then quenched to the temperature of coexistence corresponding to a given value of $\mu_{\Delta}$. After a sufficiently long period of equilibration, typically 200000 MCS's, when the system has relaxed to a thermodynamic equilibrium state, the probability distribution function $\overline{\mathcal{P}}\left(\varepsilon, x_{c}\right)$ is sampled over a period of $(10-200) \times 10^{6}$ MCS's. The method enables us to simulate systems of relatively large sizes and to study the systematic convergence towards the thermodynamic limit of the finitesize thermodynamic behavior of the physical model Hamiltonian. Though, the method is still quite time consuming. In the iterations, the system size can only be increased in very small steps; the statistics required to obtain the initial estimate of the spectral free-energy function $\mathcal{F}$ as described in step (i) is already considerable, being typically of the order of $50 \times 10^{6}$ MCS's per particle for a system of size $L=10$.

We have also performed a limited number of simulations within the canonical ensemble in order to obtain complementary results for the phase behavior of the model. The simulations based on the canonical ensemble formulation, where cholesterol concentration is one of the thermodynamic control parameters and thus fixed, are done in the following manner. The system is prepared in a high-temperature liquid state as described above, with a fixed number of cholesterol molecules randomly distributed throughout the system. The system is then quenched to the lower temperature and equilibrated over a period of 200000 MCS's and the various thermodynamical quantities are then measured over a period of $5-20 \times 10^{6}$ MCS's. In the simulations we have calculated a number of quantities including the following structure factors,

$$
\begin{aligned}
S_{T}(\vec{q})= & \frac{1}{N}\left\{\left\langle\rho_{T}(\vec{q}) \rho_{T}(-\vec{q})\right\rangle-\left\langle\rho_{T}(\vec{q})\right\rangle^{2} \delta_{\vec{q}, \overrightarrow{0}}\right\}, \\
S_{O}(\vec{q})= & \frac{1}{N}\left\{\left\langle\rho_{O}(\vec{q}) \rho_{O}(-\vec{q})\right\rangle-\left\langle\rho_{O}(\vec{q})\right\rangle^{2} \delta_{\vec{q}, \overrightarrow{0}}\right\} \\
S_{C}(\vec{q})= & \frac{1}{N}\left\{\left\langle\rho_{C}(\vec{q}) \rho_{C}(-\vec{q})\right\rangle-\left\langle\rho_{C}(\vec{q})\right\rangle^{2} \delta_{\vec{q}, \overrightarrow{0}}\right\}, \\
S_{O-C}(\vec{q})= & \frac{1}{N}\left\{\left\langle\rho_{O}(\vec{q}) \rho_{C}(-\vec{q})+\rho_{C}(\vec{q}) \rho_{O}(-\vec{q})\right\rangle\right. \\
& \left.-2\left\langle\rho_{O}(\vec{q}) \rho_{C}(\vec{q})\right\rangle \delta_{\vec{q}, 0}\right\} .
\end{aligned}
$$

Here $\rho_{T}(\vec{q})$ is the Fourier transform of the total density operator, $\rho_{T}(\vec{r}) \equiv \Sigma_{i} \delta\left(\vec{r}-\vec{r}_{i}\right)$ and $\rho_{O}(\vec{q}), \rho_{C}(\vec{q})$ is the Fourier transforms of the partial density operators $\rho_{\alpha}(\vec{r}) \equiv \sum_{i} \delta(\vec{r}$ $\left.-\vec{r}_{i}\right) \mathcal{L}_{\alpha i}$ for the lipid chains in the ordered state $(\alpha=o)$ and the cholesterol molecules $(\alpha=c)$, respectively. $\langle\cdots\rangle$ denotes a thermal average and $N$ is the number of particles. 


\section{SIMULATION RESULTS}

In this section, we describe in terms of thermodynamic phase diagrams and observables the generic thermodynamic behavior of the minimal model defined in Eq. (2), established from systematic simulations described in the preceding section. The principal thermodynamic control parameters are the temperature $T$, and either the global cholesterol concentration, $x_{c}$, or $\mu_{\Delta}$, the effective chemical potential controlling equilibrium cholesterol concentration. We define $x_{c}$ $=N_{c} / N$, where $N$ is the total number of particles in the system and $N_{c}$ is the number of cholesterol molecules. This definition is related to the molar fraction $x_{c}^{m}$ commonly used in experimental work as $x_{c}^{m}=2 x_{c} /\left(1+x_{c}\right)$, since each lipid molecule contains two chains. The rest of the parameters in the model are fixed at specific values in all simulations. For convenience, the unit for energy is chosen to be $J_{0} \equiv V_{o-o}^{l}$, which is the strength of the longer-range attraction between chains in the ordered state, as defined by Eq. (5). The unit for lengths is set by the hard-core diameter $d$. Thus, the surface pressure $\Pi$ is fixed at $\Pi d^{2} / J_{0}=6.0$. The excitation energy of the disordered state of lipid chains is chosen as $E_{d}$ $=1.303 J_{0}$, and the degeneracy of the same state $D_{d}$ is taken to be $\ln D_{d}=7.2$. The parameters defining the interaction potentials as in Eq. (4) and Eq. (5) are set at the following specific values: $V_{o-o}^{s}=1.55 J_{0}, \quad V_{o-c}^{l}=2.45 J_{0}, \quad V_{o-c}^{s}$ $=-1.75 J_{0}, \quad V_{d-c}^{l}=0.35 J_{0}, \quad V_{d-c}^{s}=-0.35 J_{0}, \quad V_{c-c}^{l}=0.5 J_{0}$, $V_{c-c}^{s}=-1.0 J_{0}, V_{o-d}^{l}=0.5 J_{0}$, and $V_{o-d}^{s}=-0.5 J_{0}$. The radius of the short-range square-well potential was $R_{0} / d=1.3$. The values of $\Pi, R_{0}, E_{d}$, and $\ln D_{d}$ are chosen so that the latent heat of the main transition and change in surface area across the main transition are comparable to that measured experimentally for the single-component DPPC bilayer system [19]. The value for $V_{o-o}^{S}$ is chosen such that the phase transition in the pure "lipid system" is located in the regime where the translational and the internal degrees of freedom are coupled (see discussion in Sec. II). Other parameters, $V_{\alpha-c}^{s, l}(\alpha=o, l, c)$ are also set at certain values, so that the phase behavior predicted by the model resembles that of the DPPC-cholesterol bilayer. Note that the values given above for the parameters are those used for the results quoted in the following. These values, however, are not unique in that the same generic phase behavior holds for a large set of different parameter values.

\section{A. Phase diagrams: simulations within the grand canonical ensemble}

In order to establish phase diagrams for our model system, we have performed simulations within the semigrand canonical ensemble, using the simulation methods described in Sec. III.

In Fig. 3 are shown the phase diagrams constructed based on our simulation data. Figure 3(a) is presented in terms of two control parameters, the global concentration of cholesterol $x_{c}$ and a reduced temperature $T / T_{\mathrm{M}}$, where $T_{\mathrm{M}}$ is the temperature of the main transition in the pure lipid system, while Fig. 3(b) is a conjugate representation in the parameter space spanned by $\mu_{\Delta}$ and $T / T_{\mathrm{M}}$. As clearly illustrated in the phase diagrams, the generic thermodynamic behavior of our model is characterized by three principal phases, an so phase,
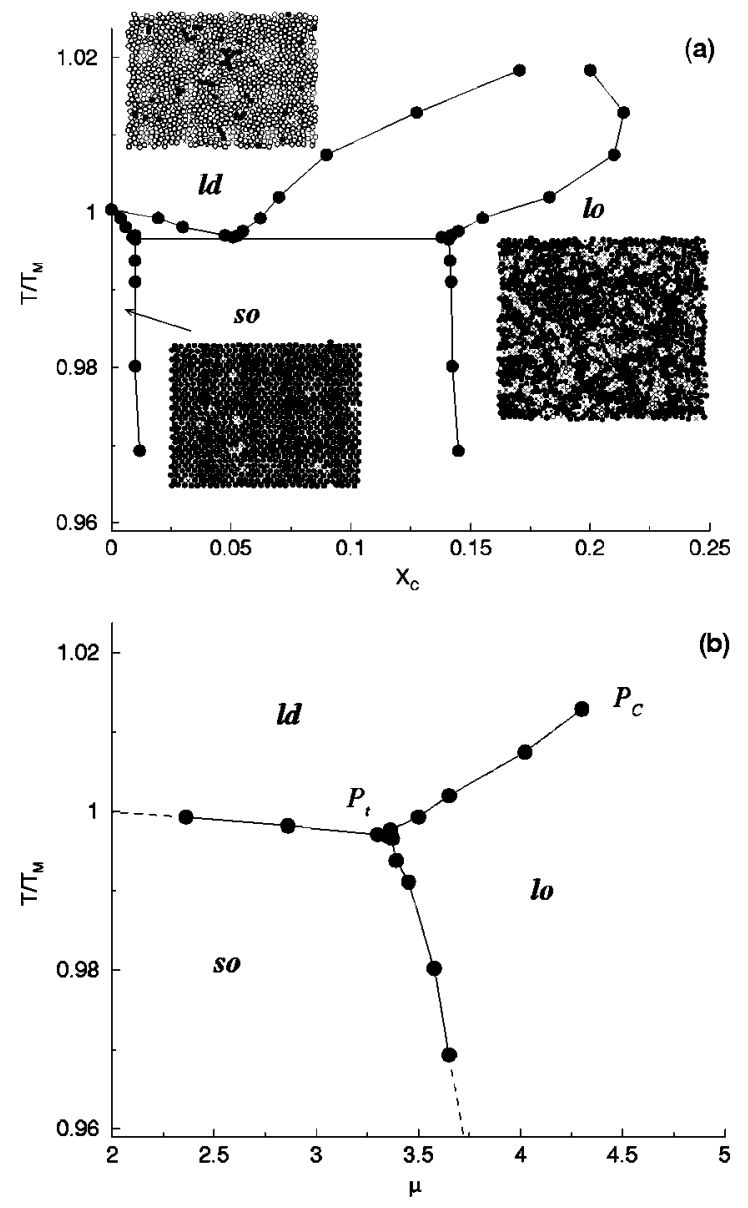

FIG. 3. Phase diagram for the lipid-cholesterol model. (a) The phase diagram as a function of cholesterol concentration $x_{\mathrm{C}}$ and reduced temperature $T / T_{\mathrm{M}}$. The insets to the figure show different snapshots of microconfigurations corresponding to the different phases in the diagram. The snapshots are not given to scale. The different phases labeled in the phase diagram are so, solid-ordered (gel); ld, liquid-disordered (fluid); and lo, liquid-ordered, where the first letter refers to the lateral order of the phase and the second letter refers to the conformational order of the phase. (b) The phase diagram as a function of the chemical potential difference $\mu_{\Delta}$ and $T / T_{\mathrm{M}} \cdot P_{\mathrm{C}}$ and $P_{\mathrm{t}}$ are the critical point terminating the ld-lo coexistence region and the triple point, respectively, as described in the text.

an ld phase, and an lo phase, as defined in the Introduction, by three first-order transitions between these three phases and finally, by two special points, a critical point, $P_{\mathrm{C}}$, which terminates the ld-lo transition and a triple point $P_{\mathrm{t}}$ at which all three phases coexist.

In Fig. 4 are shown examples of the finite-size analysis of the phase coexistence. This figure shows the spectral free energies $\mathcal{F}_{L}\left(x_{c}\right)$ calculated as a function of the system size $L$, for two of the three different cases of first-order transitions, corresponding to the so-lo and the ld-lo coexistence, respectively. In each case, a value of $T$ is chosen and kept fixed in simulations of systems of different sizes while the parameter $\mu_{\Delta}$ is tuned and a specific value $\mu_{\Delta}^{*}(T ; L)$ is determined for each system size from the "coexistence" condition-two equal-energy minima in $\mathcal{F}_{L}\left(x_{c}\right)$.

The figure shows a monotonic increase with system size $L$ in the "interfacial energy," $\Delta \mathcal{F}_{L}\left(x_{c}\right)$, defined as the height 

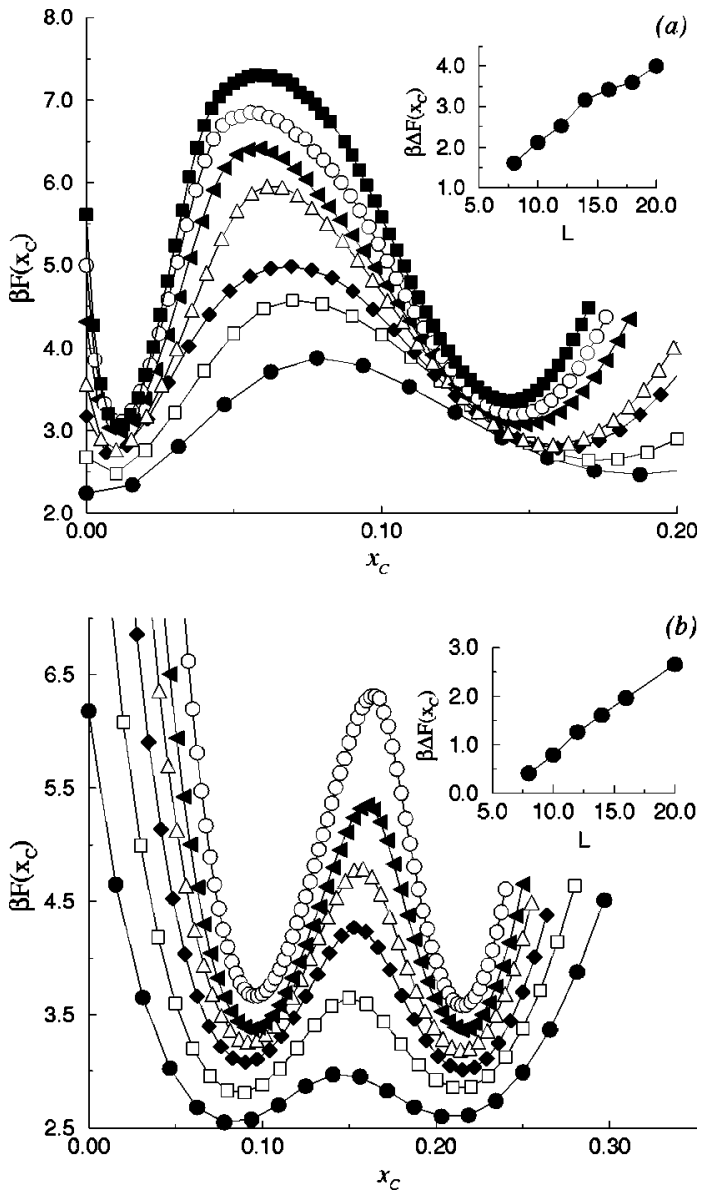

FIG. 4. Finite-size scaling plot of $\mathcal{F}_{L}\left(x_{\mathrm{C}}\right)$. (a) $T=0.969 T_{\mathrm{M}}$ (so-lo phase coexistence). The system sizes are $L=8,10,12,14$, 16, 18, and 20. (b) $T=1.007 T_{\mathrm{M}}$ (ld-lo phase coexistence). The system sizes are $L=8,10,12,14,16$, and 20 . The insets to the figures show the barrier height $\mathcal{F}\left(x_{\mathrm{C}}\right)$ as a function of system size. The lines connecting the points in the two insets are guides to the eye.

of the maximum relative to the minima of the spectral freeenergy function. This finite-size behavior indicates the existence of a first-order transition [20]. The magnitude of the energy barriers between the coexisting phases can be estimated. For example, for a system of size $L=20$, $\Delta \mathcal{F}_{L}\left(x_{c}\right)_{\mathbf{l d}-\mathrm{lo}} \simeq 3 k_{B} T$ and $\Delta \mathcal{F}_{L}\left(x_{c}\right)_{\text {so-lo }} \simeq 4.5 k_{B} T$.

The finite-size analysis also yields evidence for the presence of a critical point terminating the ld-lo coexistence. In Fig. 5 we show the finite-size analysis of the simulation data for three different temperatures close to the critical endpoint of the ld-lo coexistence region. The inserts in the figure show the variation of the spectral free energy as a function of system size for the three different temperatures. For the lowest temperature, $T=1.007 T_{\mathrm{M}}$ [Fig. 5(a)], the finite-size analysis shows a linear increase of the energy associated with an interface between the ld and the lo phases, indicating the existence of a first-order phase transition. The slope of the linear relation corresponds to the interfacial tension between the two phases. For the highest temperature, $T$ $=1.018 T_{\mathrm{M}}$ [Fig. 5(c)], $\Delta \mathcal{F}_{L}\left(x_{c}\right)_{\mathbf{l d}-\mathbf{l o}}$ decreases as a function of system size $L$, demonstrating the absence of a phase transition for this temperature. Finally, the results for $T$ $=1.013 T_{\mathrm{M}}$ [Fig. 5(b)] suggest that the "interfacial energy" at this temperature for large system sizes approaches a con-

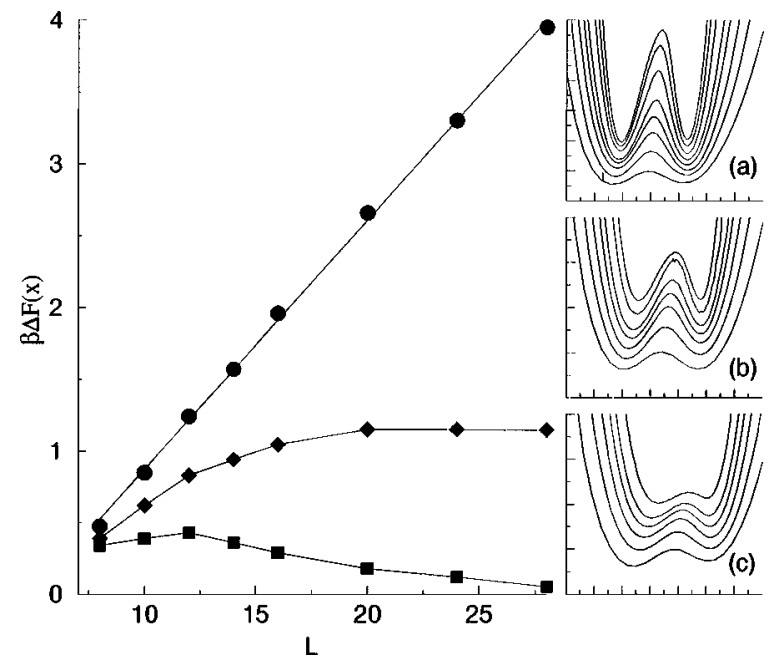

FIG. 5. Finite-size scaling plots of $\Delta \mathcal{F}_{L}\left(x_{\mathrm{C}}\right)$ at three temperatures close to the critical point of the ld-lo coexistence region. (a) $(\bullet) T=1.007 T_{\mathrm{M}},(\mathrm{b})(\diamond) T=1.013 T_{\mathrm{M}}$, and (c) $(\mathbf{\square}) T=1.018 T_{\mathrm{M}}$. The system sizes are $L=8,10,12,14,16,20,24$, and 30 . The inserts to the figure show the spectral free-energy $\mathcal{F}_{L}\left(x_{\mathrm{C}}\right)$ for the different system sizes. The line connecting the points in (a) is a linear fit to the barrier height as a function of system size. The lines connecting the points in (b) and (c) are guides to the eye.

stant value, indicating that the critical point of the ld-lo coexistence region is located close to $T=1.013 T_{\mathrm{M}}$.

A precise determination of the line of the three-phase coexistence, or the position of the triple point, requires systematic simulations for different system sizes, which turns out to be practically impossible. We have, therefore, contented ourselves with making a good estimate from a single histogram calculated for a sufficiently large system. Figure 6 shows the histogram $\mathcal{P}\left(\varepsilon, x_{c}\right)$ for a system of size $L=18$ at a temperature $0.997 T_{\mathrm{M}}$. The histogram demonstrates the coexistence of the three distinct phases: the so phase, with a low internal energy and low cholesterol concentration, the ld phase, with a high internal energy and a very modest cholesterol concentration, and the lo phase, with an intermediate internal energy and a relatively high cholesterol concentration. Based on this result we have estimated the location of the three phase line

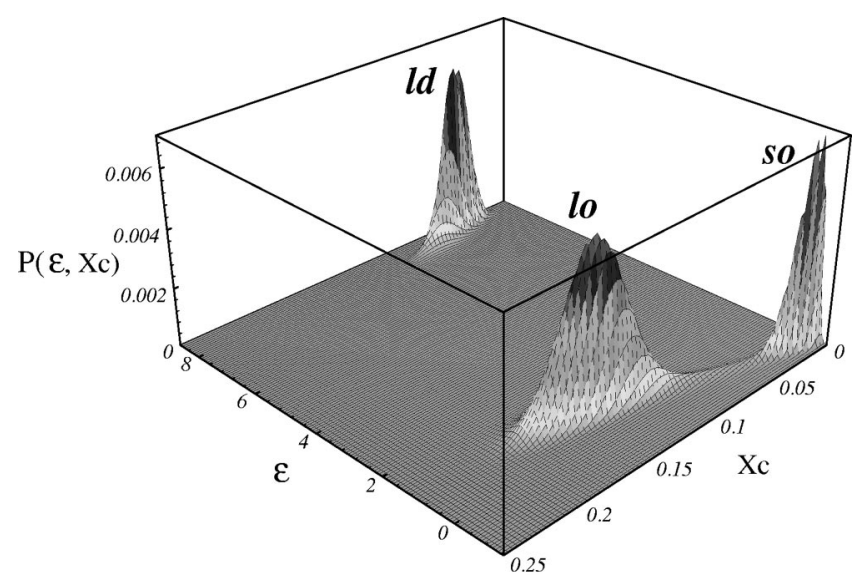

FIG. 6. The histogram $\mathcal{P}\left(\varepsilon, x_{c}\right)$ for a system size of $L=18$ and $T=0.997 T_{\mathrm{M}}$. The three coexisting phases are located at cholesterol concentrations $x_{c}=0.010(\mathbf{s o}), x_{c}=0.053$ (ld), and $x_{c}=0.143$ (lo). 
to be close to $T \simeq 0.997 T_{\mathrm{M}}$ and the concentration of cholesterol in the three coexisting phases to be, respectively, $x_{c, \text { so }}$ $\simeq 0.010, x_{c, \text { ld }} \simeq 0.053$, and $x_{c, \mathbf{l o}} \simeq 0.143$.

The region of coexistence between the so and the Id phases is very narrow and both the low-temperature bound (the three phase coexistence line) and the high-temperature bound (the main transition point of the pure lipid system) are strongly dependent on system size. It is, therefore, also very time consuming to obtain histograms of the distribution function at the coexistence, which have sufficient accuracy for a finite-size scaling analysis. We have thus used the Ferrenberg Swendsen reweighting technique [18] to estimate the locations of the boundaries of the so-ld coexistence region. Specifically, we have through the reweighting procedure extrapolated to higher temperatures the probability distribution functions obtained from the three-phase histogram.

The phase diagrams in Fig. 3 exhibit all the characteristics of macroscopic decoupling between the translational and the chain-conformational degrees of freedom: the appearance of the lo phase, and the two uncoupled transitions associated with this phase specifically, the so-lo and the ld-lo transitions. These two transitions correspond to the distinct ordering processes of the translational and the chainconformational degrees of freedom, respectively. It is clear that this phenomenon is a macroscopic consequence of the dual-natured interaction of the cholesterol molecule with the translational and chain-conformational degrees of freedom of the lipid molecules. Figure 3(a), along with our understanding of the so-ld transition in the pure lipid system, as described in Ref. [2] and summarized in Fig. 2, provides clues as to how the molecular mechanism of cholesterol is macroscopically manifested. In the one-component system of the lipid, the so-ld transition is predominantly driven by the conformational entropy associated with the disordered state of the lipid chains, and the transition temperature $T_{\mathrm{M}}$ is determined by the competition between the conformationalentropy effect and the strength of the effective interactions between conformationally ordered chains. Incorporation of the cholesterol molecule into the lipid system creates a complex picture of macroscopic ordering phenomena. Below $T_{\mathrm{M}}$, the so phase can only solubilize very low concentrations of the cholesterol without losing its (quasi-long-range) translational order, due to the "crystal-breaking" function of the cholesterol. Once the concentration reaches certain small, temperature-dependent values, the "crystal-breaking" function of cholesterol indeed causes a breakdown of the global packing order of the chains, or the loss of the long-range translational order. This breakdown in turn affects the chainordering process, as it also implies a reduction in the strength of the effective cohesive interactions between chains in the conformationally ordered state. The reduced effective interaction strength thus sets a maximal temperature for the macroscopic ordering of chain conformations; and this maximal temperature is essentially the temperature of the three-phase line. Below this temperature, the cohesive interaction between chains, albeit at its reduced strength, dominates over the conformational-entropy effect and sustains a macroscopic conformational order, even though the translational order is lost. The "chain-ordering" tendency of the cholesterol, or the high affinity of the cholesterol for conformationally ordered chains, results in relatively high solubility of the cholesterol in the lipid matrix, corresponding to the wide miscibility gap between the lipid-rich so and the cholesterolrich lo phases. In contrast, above the temperature of the three-phase coexistence, and for intermediate cholesterol concentrations, the effect of conformational entropy takes precedence over both the cohesive interactions and the chainordering effect of cholesterol, favoring the ld phase as the equilibrium phase. Under this entropy dominance, the chainordering effect of cholesterol translates into its low affinity for disordered lipid chains, as reflected in the moderate solubility of cholesterol molecules in the ld phase. At high enough cholesterol concentrations, however, the chainordering effect wins the competition over the entropy effect, reinstating the macroscopic order in the lipid chain conformations, i.e., the lo phase. This ordering event, which only involves the conformational degrees of freedom, is manifested in the reasonably wide region of the ld-lo coexistence.

\section{B. Structural analysis: simulations within the canonical ensemble}

In this section we present results of a detailed structural analysis of the different phases described above. The results have all been obtained by performing simulations within the canonical ensemble as described in Sec. III. Each simulation is performed for a system size of $N=1600$. The thermodynamic average of the structure factor contains $10^{5}$ different microconfigurations with a time interval of 100 MCS's between two consecutive configurations.

Figures 7-11 summarize calculated structure factors characterizing the lateral structures of the various thermodynamic phases. Figures 7(a) and 7(b) show, for the so and the ld phase, respectively, the structure factors $S_{T}(\vec{q})$ and $S_{O}(\vec{q})$ as well as the circular averages of $S_{T}, S_{O}, S_{C}$, and $S_{O-C}$. In Fig. 7(a) the plots of $S_{T}(\vec{q})$ and $S_{O}(\vec{q})$ give a clear signal of a solid hexagonal phase characterized by a lattice spacing close to $d$. In Fig. 7(b) the plot of $S_{T}(\vec{q})$ demonstrates the liquid characteristics of the ld phase. The average interparticle distance can be determined from the position of the first diffuse scattering ring, and a rough estimate gives a value of $\langle r\rangle \approx 1.115 d$.

Figure 8 displays both the total structure factor $S_{T}$ and the two partial structure factors $S_{O}$ and $S_{C}$, calculated at $T$ $=0.969 T_{\mathrm{M}}$ and $x_{C}=0.165$ where the system is in the lo phase. The characteristics of the phase, as far as its lateral structure is concerned, is unambiguously that of a liquid. More systematic analysis of the lo phase is summarized in Fig. 9. The three upper figures show the circular averages of $S_{T}, S_{O}, S_{C}$ and $S_{O-C}$ calculated at three different locations in the parameter region where the lo phase exists. The three lower figures are the corresponding snapshots of microconfigurations. The collective ordering in conformations of the lipid chains is apparent. The figure at the right further demonstrates the "rigidifying" effect of the cholesterol: The high degree of chain ordering as illustrated in the snapshot is stabilized only by the incorporated cholesterol, as the pure lipid system is in the ld phase at this temperature. Clearly, the appearance of the lo phase as a stable equilibrium phase is a macroscopic manifestation of the dual molecular function of the cholesterol. When present at relatively high concentrations, the cholesterol destroys the quasi-long-range 
(a)
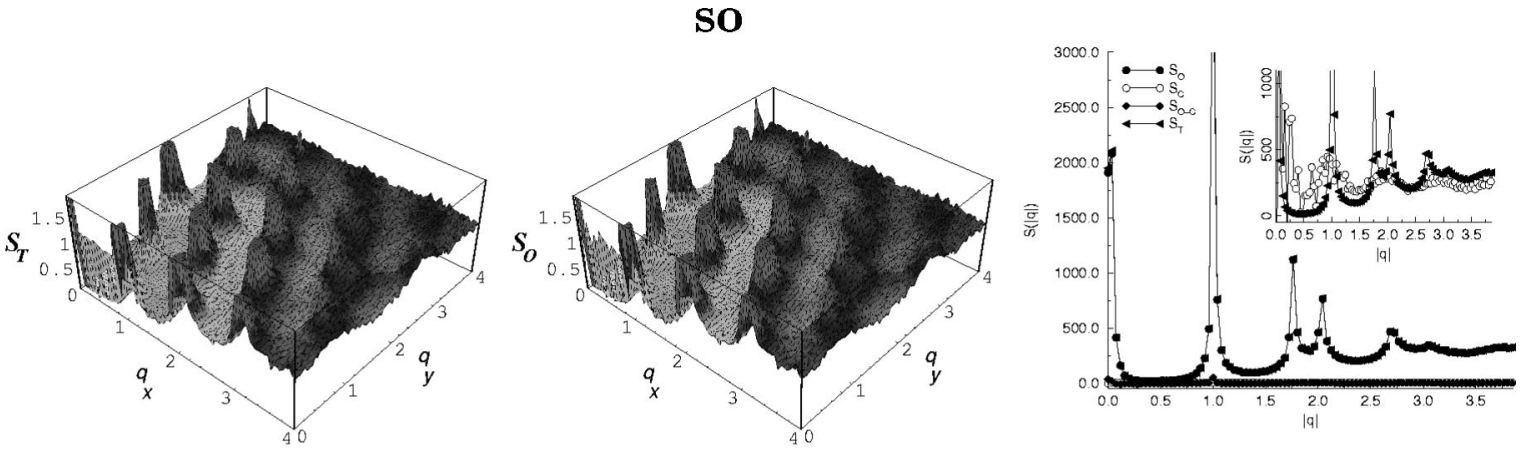

(b)
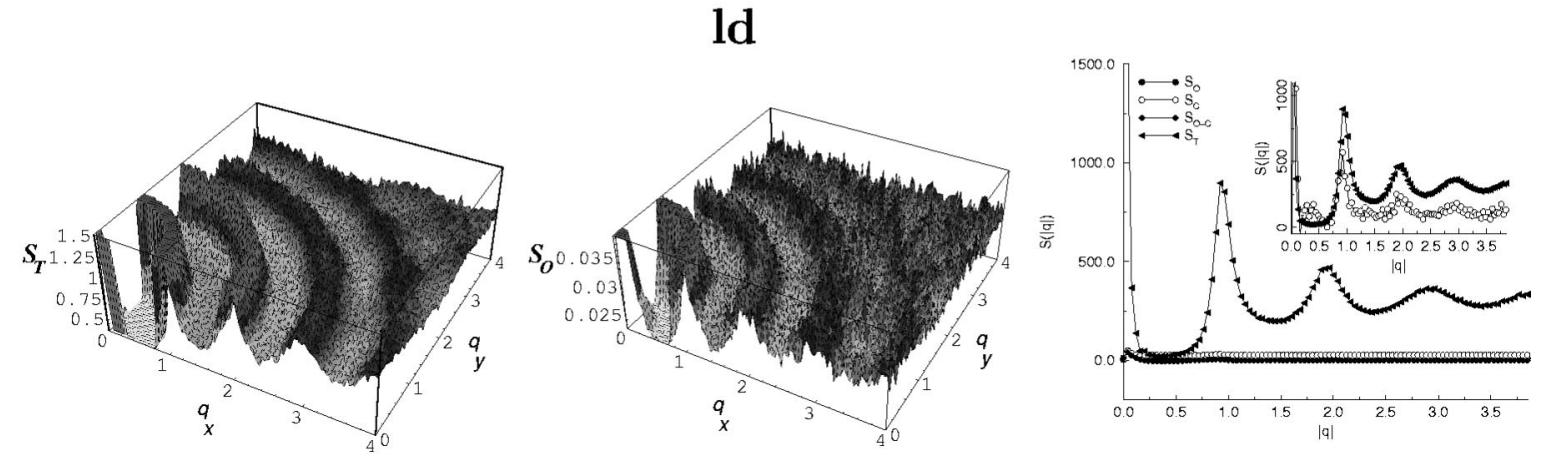

FIG. 7. The structure factors $S_{T}(\vec{q})$ and $S_{O}(\vec{q})$ calculated within the so phase and the ld phase, respectively. The three figures in (a) show the results for $T=0.969 T_{\mathrm{M}}, x_{c}=0.010$ (so phase). The three figures in (b) show results for $T=1.007 T_{\mathrm{M}}, x_{c}=0.085$ (ld phase). The left figures show $S_{T}(\vec{q})$, the middle figures show $S_{O}(\vec{q})$, and the right figures show the circular averages for $S_{T}, S_{O}, S_{C}$, and $S_{O-C}$. The $q$ values are given in units of $2 \pi / d$. The Bragg peaks in (a) characterize a hexagonal-ordered solid structure with a lattice spacing around $d$. The diffuse rings in (b) show the existence of the liquid phase. The average interparticle distance can be determined from the position of the first ring and gives a value of around $1.115 d$.

translational order and at the same time induces or reinforces macroscopic order in lipid chain conformations.

Figure 9 offers more information on the details of the lateral structure of the lo phase. The three graphs of $S_{C}(\vec{q})$ all bear a diffuse ring located close to $|q|=0.4(2 \pi / d)$. The intensity of the ring increases with the concentration of the cholesterol. An examination of the corresponding snapshots gives clue to the structures that give rise to these rings. It appears that most of the cholesterol molecules and a significant fraction of the lipid chains aggregate with their own kind to form one-dimensional threadlike microstructures. The physical reason for this particular structure, roughly speaking, lies in the difference between the interaction po- tential between two cholesterol molecules [Fig. 1(d)] and that between a cholesterol molecule and an ordered lipid chain [Fig. 1(b)]. The relatively weaker cholesterolcholesterol interaction gives the cholesterol a tendency to minimize nearest-neighbor contacts between its own kind in exchange for maximal number of contacts with ordered lipid chains. The formation of the threadlike microstructures allows that tendency to be expressed to an appreciable extent at high concentrations of the cholesterol. The data for $S_{O-C}$ indicates that the average distance between an ordered lipid chain and a cholesterol molecule is about $1.3 d$. In a threadlike structure where two cholesterol molecules are separated by one lipid chain, the distance between the cholesterol mol-

lo
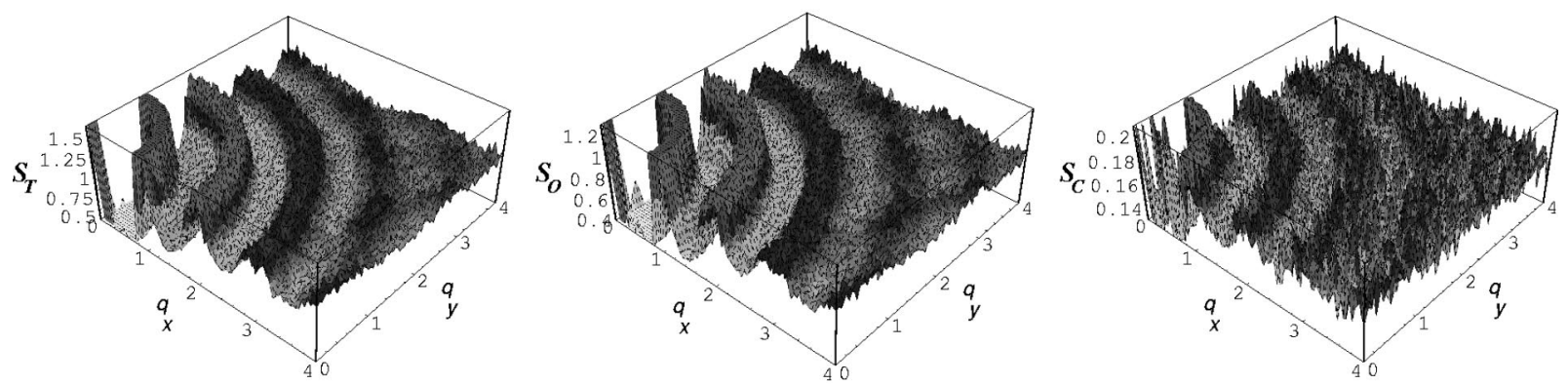

FIG. 8. The structure factors $S_{T}(\vec{q}), S_{O}(\vec{q})$, and $S_{C}(\vec{q})$ calculated at $x_{c}=0.165, T=0.969 T_{\mathrm{M}}$ within the lo phase. The $q$ values are given in units of $2 \pi / d$. 


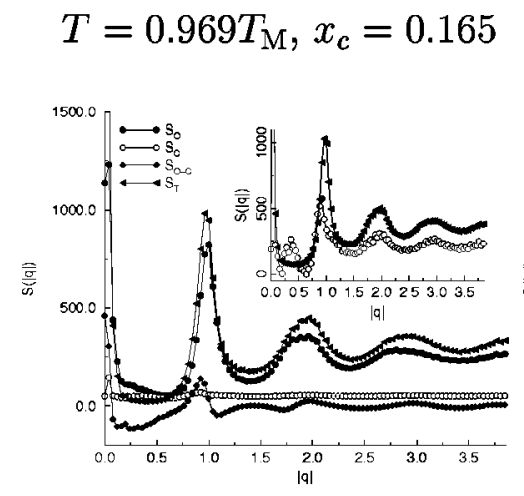

$$
T=0.969 T_{\mathrm{M}}, x_{c}=0.25
$$$$
T=1.007 T_{\mathrm{M}}, x_{c}=0.25
$$
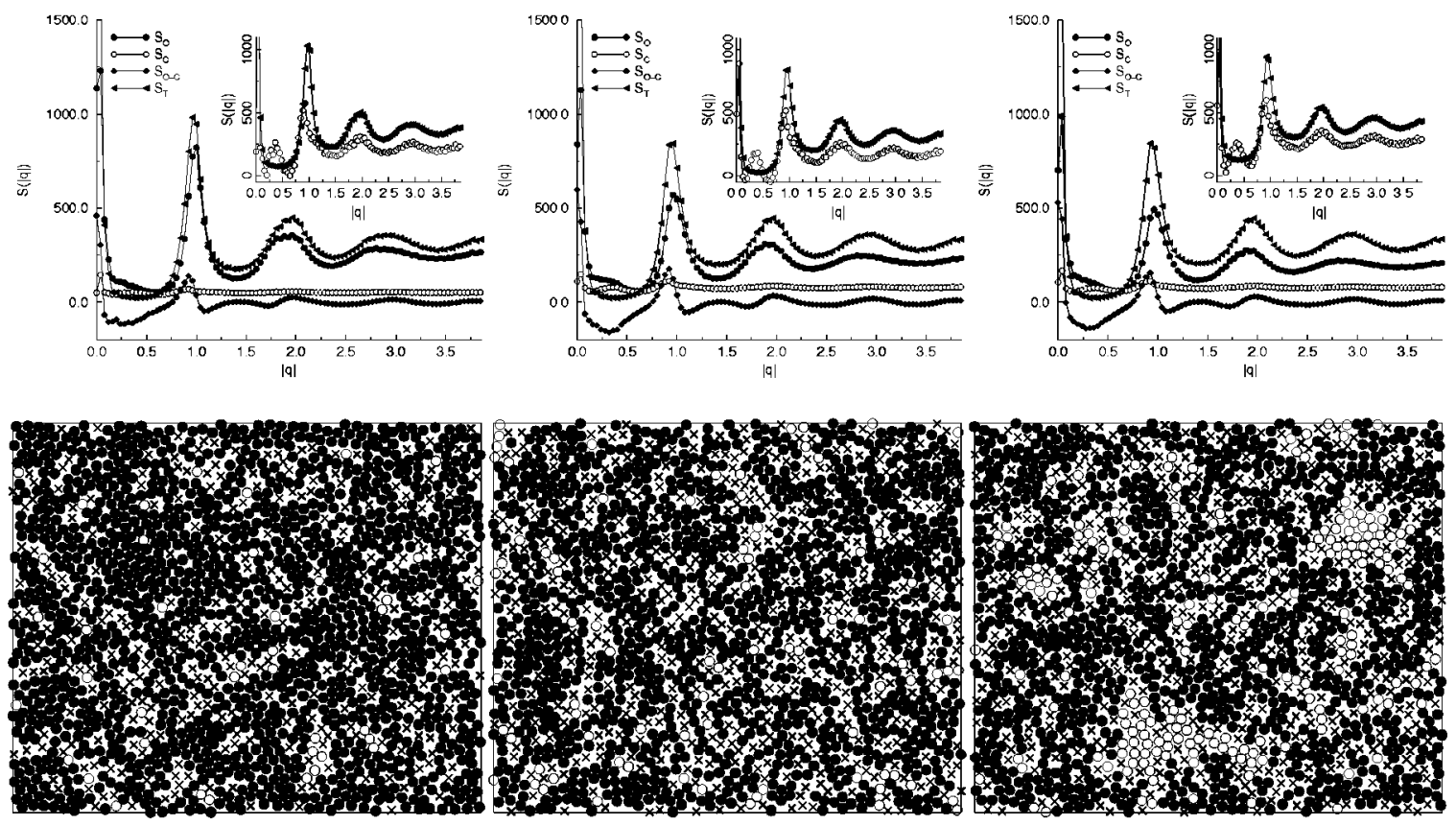

FIG. 9. The structure factor calculated at different locations within the lo phase. The upper three figures show the circular averages of $S_{T}$, $S_{O}, S_{C}$, and $S_{O-C}$ calculated at (from the left) $x_{c}=0.165, T=0.969 T_{\mathrm{M}} ; x_{c}=0.25, T=0.969 T_{\mathrm{M}}$; and $x_{c}=0.25, T=1.007 T_{\mathrm{M}}$. The $q$ values are given in units of $2 \pi / d$. The lower three figures show snapshots of the microconfigurations of the corresponding phases. In the snapshots, a lipid chain in the ordered state is shown as $(\mathbf{O})$, a lipid chain in the disordered state as $(\bigcirc)$, and a cholesterol molecule as $(\times)$.

ecules is then about 2.6d. Such a configuration should give rise to a diffuse ring located at $|q| \approx 0.4(2 \pi / d)$. And this signal should be more appreciable in $S_{C}(\vec{q})$ than in $S_{O}(\vec{q})$ naturally, due to the relatively higher fraction of those cholesterol molecules forming the threadlike structure out of the total number of the cholesterol molecules. This picture is consistent with the simulation results.

Finally Figs. 10 and 11 show the structure factors calculated within the so-lo and ld-lo coexistence region, respectively. The structure factor $S_{T}(\vec{q})$ in Fig. 10 gives a clear signal of both a liquid and a solid phase. $S_{C}(\vec{q})$, the partial structure factor related to the cholesterol, on the other hand, displays only the diffuse properties of a liquid, indicating that the cholesterol dissolves in the liquid (lo) domain. The structure factors in Fig. 11, corresponding to the ld-lo coexistence, show no sign of structural differences between the two coexisting phases. This result further supports the characterization of the lo phase as a liquid phase.

\section{DISCUSSION}

Experimentally observed equilibrium phase behavior of bilayer systems of binary mixtures of phospholipids and cholesterol is characterized by a macroscopic decoupling of the translational degrees of freedom from the internal molecular,
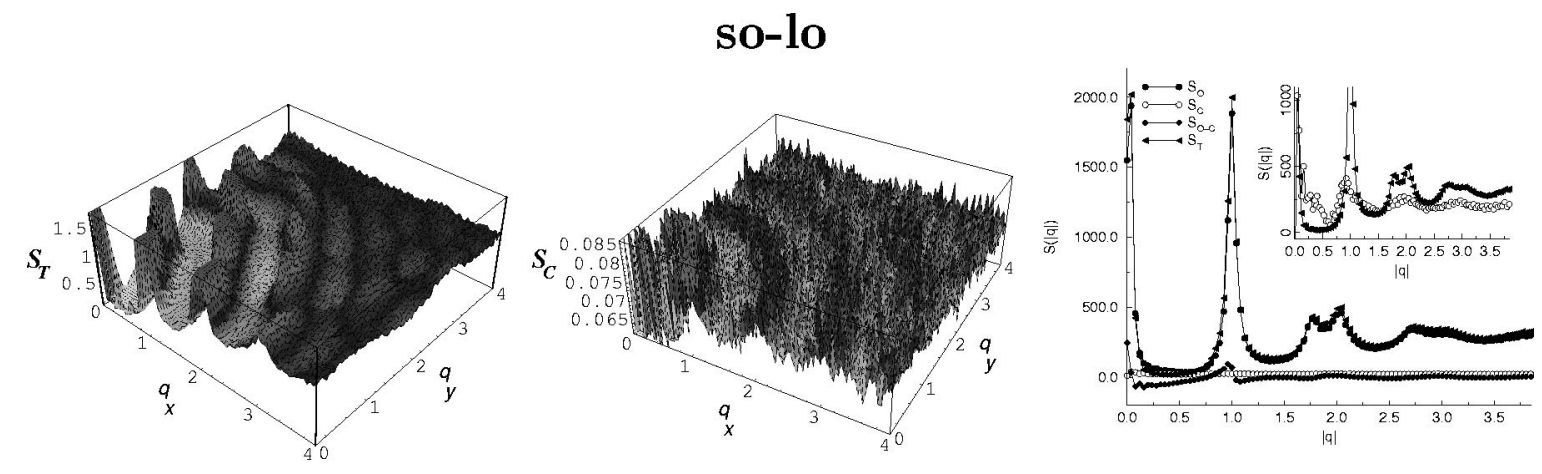

FIG. 10. The structure factors $S_{T}(\vec{q})$ (the left figure) and $S_{C}(\vec{q})$ (the middle figure) calculated at $x_{c}=0.075$ and $T=0.969 T_{\mathrm{M}}$ within the region of phase coexistence of the so and the lo phases. The figure at the right gives the circular averages of $S_{T}, S_{O}, S_{C}$, and $S_{O-C}$. The $q$ values are given in units of $2 \pi / d$. 


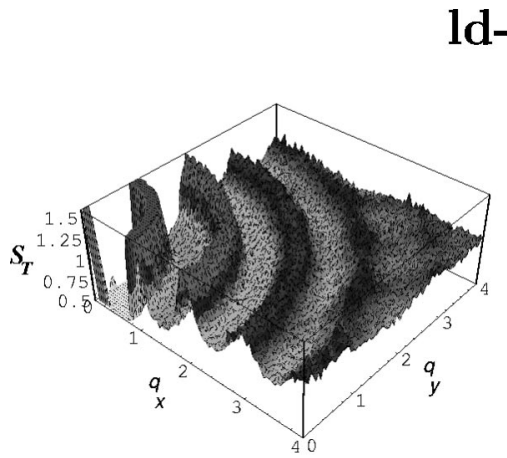

ld-lo

specifically, chain conformational, degrees of freedom. This phenomenon provides a meaningful context for investigating the generic physics involved in interplay, both at the microscopic level and at the macroscopic level, between translational and internal molecular degrees of freedom. To this end, we have formulated a microscopic model to describe a two-dimensional system composed of two distinct types of "model molecules," representing lipid molecules and cholesterol molecules, respectively, and we have carried out a statistical mechanical study, based on Monte Carlo computer simulations, of the model to investigate the equilibrium phase behavior of the model system.

The model differs in an essential way from some of the earlier lattice models developed to describe the phase behavior of lipid-cholesterol mixtures: It provides an off-lattice, and therefore realistic, representation of the translational degrees of freedom in terms of a specific random-lattice algorithm. Naturally, this feature enables us to characterize (and make predictions on) the macroscopic behavior of the translational degrees of freedom, i.e., the lateral structures of different thermodynamic phases of the model system in a faithful way.

Given both our aim of elucidating generic behavior of the interplay and the considerable computational effort required by the explicit treatment of translational degrees of freedom, the rest of the ingredients of the model correspond to minimal descriptions of the relevant microscopic physics. The chain conformational degrees of freedom and the associated phase space are simplified into two molecular conformational states. Cholesterol is treated as a simple substitutional impurity. Its molecular function in a lipid bilayer is described, based on an earlier hypothesis [5,21], to be dual, both as a "crystal breaker" and as a "chain rigidifier." This molecular mechanism forms a microscopic basis for the interplay between translational and conformational degrees of freedom. The microscopic interactions, those between a cholesterol molecule and a lipid chain, in particular, are all designed to only contain features that are essential and necessary for describing both the simple phase behavior of onecomponent systems of lipid and the dual molecular function of cholesterol.

Our rather extensive and systematic study of the minimal model based on Monte Carlo computer simulations has led to a phase diagram for the model system. This phase diagram displays the same topology as the experimentally obtained phase diagram [10]. Particularly, the theoretical phase diagram demonstrates that the proposed microscopic model does provide a picture of microscopic physics underlying the ability of cholesterol to uncouple, already at very low con-
FIG. 11. The structure factors calculated at $x_{c}=0.17$ and $T=1.010 T_{\mathrm{M}}$ within the region of phase coexistence of the ld and the lo phases. The left figure shows $S_{T}(\vec{q})$, the right figure gives the circular averages of $S_{T}, S_{O}, S_{C}$, and $S_{O-C}$. The $q$ values are given in units of $2 \pi / d$. centrations, the macroscopic ordering processes of the translational and chain conformational degrees of freedom. The fact that the model is only a minimal one suggests that the macroscopic decoupling between translational and chain conformational degrees of freedom in these systems may not depend sensitively on details of the microscopic physics and may, therefore, be a generic phenomenon. It has actually been argued that the phase behavior of the DPPC-cholesterol bilayer system contains some generic features for mixtures of lipids and cholesterol or cholesterol-like molecules [5,22,23], although more concrete experimental evidence is yet to be obtained.

In addition, our paper provides detailed information on the lateral structure of the different phases. The structure of the cholesterol-rich phase is of particular interest, since it has been the subject of a long and continuing debate in the experimental literature [24]. The structure factor that we have obtained from the simulations clearly shows that the lo phase is indeed a liquid phase. More interestingly, the partial structure factors associated with cholesterol molecules and ordered lipid chains, respectively, show signatures of an additional structure that is characterized by length scales roughly twice that of the average distance between nearest neighbors. Inspection of the related microscopic configurations leads to an interesting observation: cholesterol molecules and those lipid chains that are in direct contact with cholesterol molecules tend to form "threadlike"' structures. In other words, in these structures, each molecule tends to align (linearly) with its own kind. This tendency becomes more pronounced at higher cholesterol concentrations (data not shown in the paper). The "threads" are short, involving only a few molecules, and there is no sign of long-range correlations in their orientations. The origin of this behavior may be understood as follows. At low and intermediate temperatures (compared with the temperature of main transition in the onecomponent system of lipid), the system in the lo phase consists of cholesterol and chains in the ordered state only. Therefore, the model effectively becomes an off-lattice antiferromagnetic Ising model with a spin-exchange interaction of strength $\frac{1}{4} V_{o-o}\left(R_{i j}\right)+\frac{1}{4} V_{c-c}\left(R_{i j}\right)-\frac{1}{2} V_{o-c}\left(R_{i j}\right)$ and an external field [25]. This model has not been studied. But, a related model, an antiferromagnetic Ising model defined on a lattice with elastic deformability, has been shown to display a low-temperature stripe phase, where like-signed spins line up to form stripes [26]. The characteristic periodicity in the stripe phase is twice that of the average lattice spacing [26].

The presence of the "threadlike" structures revealed by 
our simulations has some experimental support, particularly from early fluorescence [27] and x-ray diffraction [28] studies. The results of these studies were interpreted as support for a structural model where lipid and cholesterol molecules formed stoichiometrical complexes and moreover, the complexes aligned themselves linearly (see [29] for an early review of those studies). Our study shows, however, that the partial alignment of molecules as observed in the simulations requires no chemically specific mechanisms such as the formation of stoichiometrical complexes. It is important to note that the "threadlike" structures in the lo phase are not the ripple structures observed in experimental systems in so-lo coexistence region [30]. The ripple structures involve much larger length scales as well as nonplanar membrane surface configurations $[31,32]$.

We end our presentation with a final remark on further applications of the proposed minimal model. Indeed, the topology of the presented phase diagram holds only for a range of values of the microscopic parameters. It is expected that the model, given its various microscopic parameters, is capable of describing more types of equilibrium phase behavior than that discussed in this paper. In other words, by systematically varying the values of certain parameters and carrying out similar studies of the model, one can establish a much larger picture of generic equilibrium phase behavior of two-component systems. Varying values of the microscopic parameters may represent variations in, for example, sterol type. In fact, cholesterol is the end product of a biosynthetic pathway, along which there are other sterols acting as evolutionary precursors to cholesterol. Further studies of the model are in progress, aiming at capturing the systematics of equilibrium phase behavior of a range of two-component systems modeling mixtures of lipids with those sterols along the biosynthetic pathway.

\section{ACKNOWLEDGMENTS}

This work was supported by the Danish Natural Science Research Council, the Danish Technical Research Council, le FCAR du Quebec by center and team grants, and by the NSERC of Canada by operating and equipment grants. Both M.N. and L.M. acknowledge support from the Danish Research Academy. M.J.Z. and O.G.M. are associates of the Canadian Institute of Advanced Research under the program on the Science of Soft Surfaces and Interfaces. We are grateful to Jens Risbo for sharing with us his expertise on implementing the simulation techniques involving modified Hamiltonians. We wish to thank Myer Bloom and Jenifer Thewalt for very interesting discussions.
[1] Handbook of Biological Physics, edited by R. Lipowsky and E. Sackmann (North-Holland, Amsterdam, 1995), Vol. 1A-B.

[2] M. Nielsen, L. Miao, J. H. Ipsen, K. Jørgensen, M. J. Zuckermann, and O. G. Mouritsen, Phys. Rev. E 54, 6889 (1996).

[3] O. G. Mouritsen, Chem. Phys. Lipids 57, 179 (1991).

[4] O. G. Mouritsen and M. J. Zuckermann, Chem. Phys. Lett. 135, 294 (1987).

[5] J. H. Ipsen, G. Karlström, K. Wennerström, and M. J. Zuckermann, Biochim. Biophys. Acta 905, 162 (1987).

[6] J. Lemmich, K. Mortensen, J. H. Ipsen, T. Hønger, R. Bauer, and O. G. Mouritsen, Eur. Biophys. J. 25, 293 (1997).

[7] C. D. Mitchell and B. J. Litman, Biophys. J. 75, 896 (1998).

[8] S. Halstenberg, T. Heimburg, T. Hianik, U. Kaatze, and R. Krivanek, Biophys. J. 75, 264 (1998).

[9] D. Needham, T. J. McIntosh, and E. Evans, Biochemistry 27, 4668 (1988).

[10] M. Vist and J. H. Davis, Biochemistry 29, 451 (1990).

[11] D. A. Pink, T. J. Green, and D. Chapman, Biochemistry 19, 349 (1980).

[12] P. S. Sahni, G. S. Grest, and M. P. Anderson, Phys. Rev. Lett. 50, 263 (1983).

[13] S. Doniach, J. Chem. Phys. 68, 4912 (1978).

[14] N. Metropolis, A. Rosenbluth, M. Rosenbluth, A. Teller, and E. Teller, J. Chem. Phys. 21, 1087 (1953).

[15] D. Frenkel and B. Smit, Understanding Molecular Simulations (Academic Press, New York, 1996).

[16] B. A. Berg and T. Neuhaus, Phys. Rev. Lett. 68, 9 (1992).

[17] J. Risbo, Ph.D. thesis, Technical University of Denmark, 1997; J. Risbo, G. Besold, and O. G. Mouritsen (unpublished).

[18] A. M. Ferrenberg and R. H. Swendsen, Phys. Rev. Lett. 61, 2635 (1988).
[19] Phospholipids Handbook, edited by G. Cevc (Dekker, New York, 1993).

[20] J. Lee and J. L. Kosterlitz, Phys. Rev. B 43, 3265 (1991).

[21] J. H. Ipsen, O. G. Mouritsen, and M. J. Zuckermann, J. Chem. Phys. 91, 1855 (1989); Biophys. J. 56, 661 (1990).

[22] J. L. Thewalt and M. Bloom, Biophys. J. 63, 1176 (1992).

[23] F. M. Linseisen, J. L. Thewalt, M. Bloom, and T. M. Bayerl, Chem. Phys. Lipids 65, 141 (1993).

[24] Cholesterol in Membrane Models, edited by Leonard Finegold (CRL Press, Boca Raton, FL, 1993).

[25] In the case where the lo phase consists of only cholesterol and gel chains in the ordered state, the microscopic Hamiltonian Eq. (2) can be rewritten as an Ising model in an external field. If we associate the Ising spin $S=+1$ state with a chain in the ordered state and the Ising spin $S=-1$ state with a cholesterol molecule, the occupation variables $\mathcal{L}_{i o}$ and $\mathcal{L}_{i c}$ can be expressed as $\mathcal{L}_{i o}=\left(1+S_{i}\right) / 2, \mathcal{L}_{i c}=\left(1-S_{i}\right) / 2$. Rewriting Eq. (2) in terms of the Ising spin variable we get $H=\Sigma_{\langle i<j\rangle}\left(V_{o-o} / 4\right.$ $\left.+V_{c-c} / 4-V_{o-c} / 2\right) S_{i} S_{j}+\sum_{i} h(T, P) S_{i}$. Noting that the interaction potentials $V_{o-o}$ and $V_{o-c}$ have very similar strength and that the strength of the interaction potential $V_{c-c}$ is relatively small, we see that the model becomes an antiferromagnetic Ising model in an external temperature and pressure dependent field. ( $V_{o-o}, V_{o-c}$, and $V_{c-c}$ are all attractive interaction potentials.)

[26] L. Gu, B. Chakraborty, P. L. Garrido, M. Phani, and J. L. Lebowitz, Phys. Rev. B 53, 11985 (1996).

[27] J. Rogers, A. G. Lee, and D. C. Wilton, Biochim. Biophys. Acta 552, 23 (1979).

[28] S. W. Hui and Neng-Bo He, Biochemistry 22, 1159 (1983).

[29] F. T. Presti, in Membrane Fluidity in Biology, edited by R. C. 
Aloia and J. M. Boggs (Academic Press, New York, 1985), Vol. 4, pp. 97-146.

[30] K. Mortensen, W. Pfeiffer, E. Sackmann, and W. Knoll, Biochim. Biophys. Acta 945, 221 (1988).

[31] Note that bilayers in our model are considered to be flat. An interpretation of ripple structures has recently been given in terms of a mesoscopic phenomenological model for lipid bilayers [32].

[32] P. L. Hansen, L. Miao, and J. H. Ipsen, Phys. Rev. E 58, 2311 (1998). 